\title{
The effects of performing a one-legged bridge with use of a sling on trunk and gluteal muscle activation
}

\author{
Minkwon Cho ${ }^{\mathrm{a}}$, Jongwoo Bak ${ }^{\mathrm{a}}$, Yijung Chung ${ }^{\mathrm{b}}$ \\ ${ }^{a}$ Department of Physical Therapy, The Graduate School, Sahmyook University, Seoul, Republic of Korea \\ ${ }^{b}$ Department of Physical Therapy, College of Health and Welfare, Sahmyook university, Seoul, Republic of Korea
}

\begin{abstract}
Objective: The purpose of this study was to compare the activation of trunk and gluteal muscles during bridge exercises with a sling (BS), single-legged bridge exercise with a sling (SBS), single-legged bridge exercise (SB), and general bridge exercise (GB). Design: Cross-sectional study.

Methods: Twenty-five healthy participants (19 males and 6 females, aged 27.8 [4.78]) voluntarily participated in this study. In the bridging exercise, each subject lifted their pelvis with their legs and feet in contact with the sling or normal surface. The electrical activities of the erector spinae (ES), gluteus maximus (GM), external oblique (EO), and internal oblique (IO) muscles during the bridging exercises on the 2 surfaces were measured using surface electromyography. Subjects practiced each of the four bridge condition three times in random order and average values were obtained.
\end{abstract}

Results: On the ipsilateral side, activities of the IO, EO, and ES during SBS was significantly higher than those during BS, SB, and GB $(p<0.05)$. Activities of the IO and EO during SB was significantly higher than those during BS and GB $(p<0.05)$. On the contralateral side, activities of the GM and EO during SB and SBS was significantly higher than that during BS and GB ( $p<0.05$ ). These results verify the theory that the use of sling and single leg lift increases the activation trunk and gluteal muscles during bridging exercises.

Conclusions: The single-legged bridge exercise with a sling can be recommended as an effective method to facilitate trunk and gluteal muscle activities.

Key Words: Electromyography, Exercise, Muscle activation

\section{Introduction}

Stabilization exercises are performed in the presence of postural instability to enable the ability to make postural adjustments and maintain a neutral posture both consciously and unconsciously [1].

Rather than activation of specific muscles, stabilization occurs through the coordination of the entire trunk muscles [2]. The purpose of stabilization is to restore balance and ability to control movement by promoting and strengthening the stability of the spinal and pelvic muscles [3]. Trunk muscles contribute substantially to providing stability during performance of exercise, activities of daily living, and func- tional activities [4]. Since trunk muscles are necessary in maintaining various postures for daily life activities, maintenance of trunk muscle strength and endurance is highly essential [5]. Stabilization through the use of trunk muscles may be defined as the over stability and local stabilization. In order to move the trunk and the lower extremities, the surface muscles (rectus abdominus, spinal muscles, external oblique [EO]) and grossglobal muscles must be mobilized, and afterwards, the deep core muscles (internal oblique [IO], transverse abdominus, multifidus [MF]) must be mobilized along with the local muscles. A stable core provides stability during lumbar spine movement, which is essential for overall body movement and making postural adjustments [6].

Received: 14 April, 2016 Revised: 19 May, 2016 Accepted: 25 May, 2016

Corresponding author: Yijung Chung

Department of Physical Therapy, The Graduate School of Sahmyook University, 815 Hwarang-ro, Nowon-gu, Seoul 01795, Republic of Korea Tel: 82-2-3399-1639 Fax: 82-2-3399-1639 E-mail: yijung36@syu.ac.kr

(c) This is an Open-Access article distributed under the terms of the Creative Commons Attribution Non-Commercial License (http://creativecommons.org/licens es/by-nc/4.0) which permits unrestricted non-commercial use, distribution, and reproduction in any medium, provided the original work is properly cited.

Copyright $@ 2016$ Korean Academy of Physical Therapy Rehabilitation Science 
The supine position is appropriate for treatment of primary or secondary injuries to the trunk and limbs [7]. Bridge exercises are considered as a basic type of exercise performed on a mat that promotes stabilization to the trunk, reduces pressure onto the buttocks, promotes bed mobility, assists in use of bedpans and lower extremity dressing, improves ability to perform sit to stand, and facilitates movement of the pelvis, which is all considered to be not only useful for walking, but is significantly relevant to pelvic and functional movement [8]. Bridge exercises are used to prevent injury to the muscles, joints, ligaments, and tissues of the spine during daily living activities, and are considered a method of promoting stability [9]. In addition, it allows weight-bearing to occur through bilateral lower extremities, improves the ability to perform sit-to-stand, strengthens, lower vertebrae and hip extensors in preparation for the stance phase of gait [10].

Closed chain strengthening exercises for the trunk and lower extremities are not only used to activate the hip muscles, but it also activates all trunk and lower extremity muscles, allowing appropriate amount of muscle tension to occur and has an antagonistic advantage [11].

In order to increase the activation of stabilizing muscles, closed chain exercises with a sling can be performed [12], and sling exercises are considered to be effective for promoting strength, neuromuscular control, and stabilization that can be performed with ease [9].

It has been reported in the literature that trunk muscle activation increases with stabilization exercises using a cushion or sling to provide an unstable surface [13-15], proprioceptive sense and strength are also increased by performing closed chain exercises with use a sling in low back patients [16]. In addition, a greater increase in MF and erector spinae (ES) muscle activity has been reported during bridge exercise performance with use of a sling compared to performance of bridge exercise with use of a ball or a general bridge exercise (GB) on the floor [17]. Previous studies have mostly focused on investigating trunk muscle activation during

Table 1. General characteristics of the subjects

$(\mathrm{N}=25)$

\begin{tabular}{lc}
\hline Characteristic & Subjects \\
\hline Sex (male/female) & $25(16 / 9)$ \\
Age $(\mathrm{y})$ & $27.81(4.78)$ \\
Height $(\mathrm{cm})$ & $171.04(7.32)$ \\
Weight $(\mathrm{kg})$ & $65.82(12.49)$ \\
\hline
\end{tabular}

Values are presented as mean (SD). bridge exercises. Therefore, the purpose of this study was not only to determine the effects of performing bridge exercises with use of a sling on trunk and lower muscle activation, but also to investigate for which type of bridge exercise would most efficiently activate the trunk and lower extremity muscles in healthy subjects.

\section{Methods}

\section{Subjects}

This was a cross-sectional study in which twenty-five healthy subjects between the ages of 20 and 30 years old agreed to participate in the study after being informed of the study purpose, method, result, and application. Muscle activation was measured while performing bridge exercises with a sling (BS), single-legged bridge exercise with a sling (SBS), single-legged bridge exercise (SB), and the GB.

Subject who had experienced back pain, deformity of the limbs, severe orthopaedic or neurological diseases within the past 6 months were excluded from the study (Table 1).

\section{Procedures}

The maximum voluntary isometric contraction (MVIC) was obtained from the ES, EO, IO, and gluteus maximus (GM). Prior to the experiment, subjects were informed of the bridge exercise performance methods by the researcher and were allowed to practice each bridge condition three times. Each bridge condition was performed three times in random order. A description of the bridging conditions are as follows:

\section{GB}

Patients were positioned in a supine hook-lying position with 90 degrees of knee flexion. Patients were instructed to lift their hips while maintaining it in 0 degrees $[8,18]$ (Figure 1).

\section{$S B$}

Starting from the same position as the GB patients were instructed to maintain the left knee (contralateral side) in 90 degrees of flexion and to lift the right lower extremity and pelvis while maintaining the right hip in 0 degrees (neutral position) (Figure 2).

\section{$B S$}

Starting from the same position as the GB, bilateral lower extremities were placed in a sling at the level of the ankles, and were instructed to lift the pelvis while maintaining the 


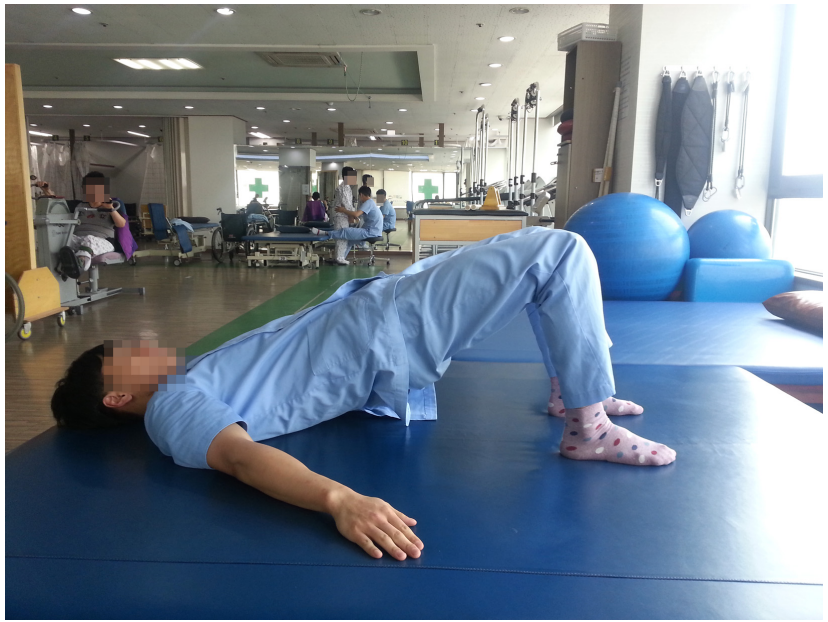

Figure 1. General bridge exercise.

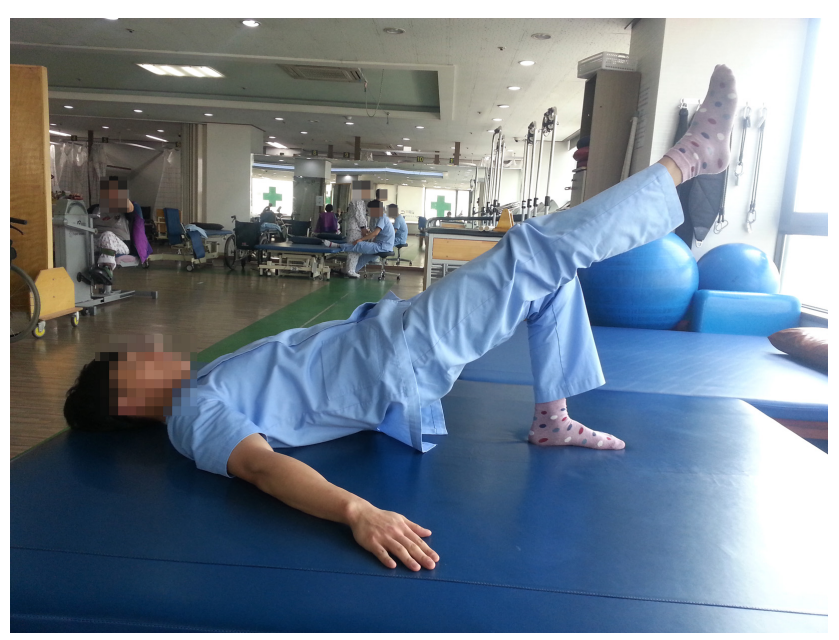

Figure 2. Single-legged bridge exercise.

hip in 0 degrees (Figure 3).

\section{SBS}

Starting from the same position as the GB, the left lower extremity was placed in a sling at the level of the ankle while the right lower extremity was used to raise the pelvis and maintain the hip in 0 degrees (Figure 4).

At the start of a signal, all subjects were instructed to lift the hip and maintain it in 0 degrees so that that the spine and pelvis were aligned, and to maintain the position for 5 seconds. Each condition was performed in random order and was repeated and measured three times with a rest period of 1 minute provided between each condition. The Telemyo 2400 G2 Telemetry electromyography system (Noraxon Inc., Scottsdale, AZ, USA, 2011) was used to examine the

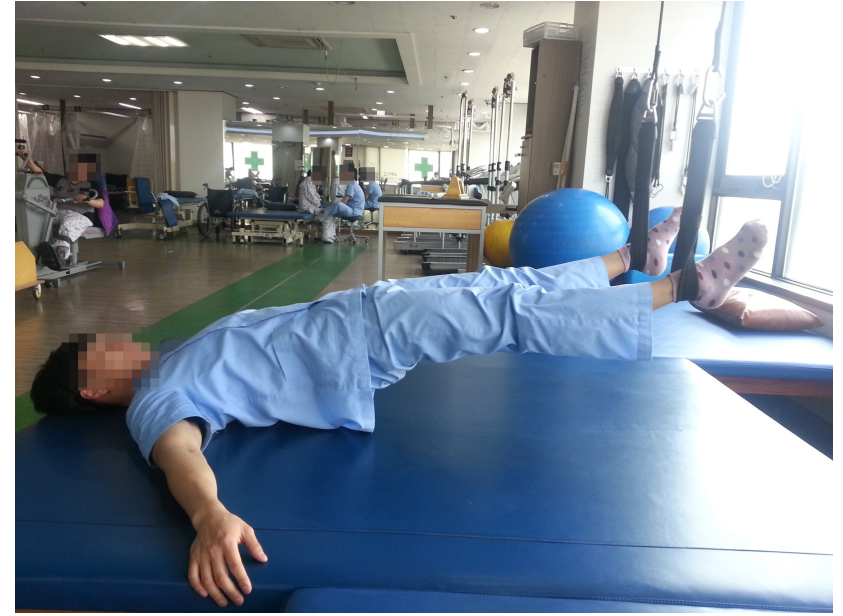

Figure 3. Bridge exercise with a sling.

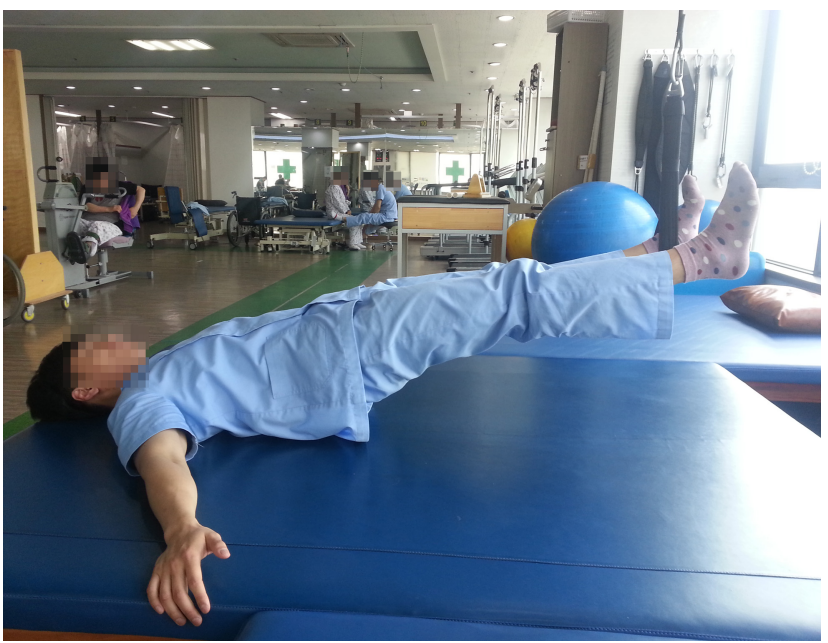

Figure 4. Single-legged bridge exercise with a sling.

muscle activation patterns during the bridge exercises. Data was measured using a sampling rate of $100 \mathrm{~Hz}$ and a band-pass filter of 10-450 Hz.

Adhesive electrodes were attached onto the $\mathrm{L} 2$ area $2 \mathrm{~cm}$ inward towards the triangle for the ES, $15 \mathrm{~cm}$ above the umbilicus for the EO, between the gluteal line and greater trochanter for the GM, and $2 \mathrm{~cm}$ downward and inward from the anterior superior iliac spine for the IO [19]. Prior to the experiment, the MVIC data analysis of the ES, EO, IO, and GM was conducted. The MVIC of the ES was assessed with the subject lying in prone position and raising the trunk off the treatment table while a therapist stabilized the lower extremities. The MVIC of the IO and EO was assessed with the subject in supine position and lifting the trunk in a diagonal direction while a therapist stabilized the lower extremities. 
The MVIC of the GM was assessed with the subject in prone position and raising both lower extremities while a therapist stabilized the upper extremities [20]. This study was approved by the Institutional Review Board at Sahmyook University (IRB no. 2-1040781-AB-N-01-2016010HR).

\section{Data and statistical analysis}

EMG measurement of muscle activity was performed using the Myoresearch XP Master Edition software (Noraxon Inc., 2011). After the data was analysed with full wave rectification and calculated to root mean square, the MVIC was normalized to a percentage value. As soon as the hip and

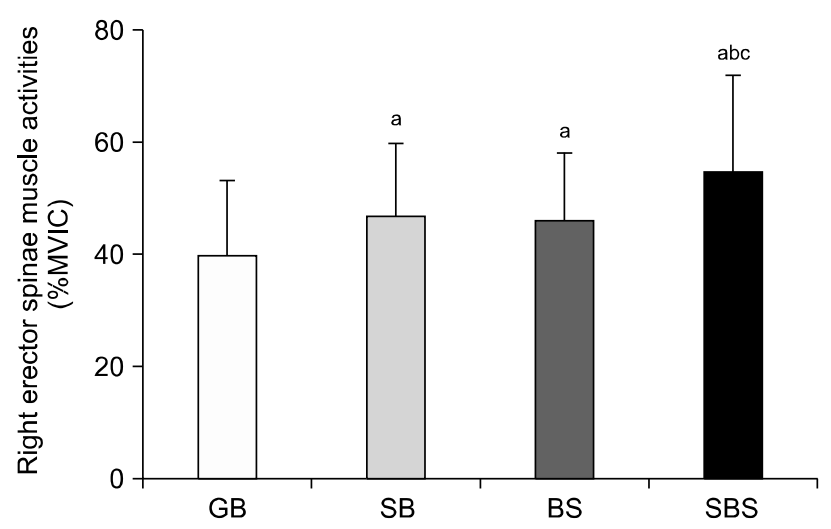

Figure 5. Right erector spinae muscle activities. GB: general bridgeexercise, SB: single-legged bridge exercise, BS: bridge exercises with a sling, SBS: single-legged bridge exercise with a sling. ${ }^{\text {a }}$ Statistically significant difference with GB $(p<0.05)$. ${ }^{\mathrm{b}}$ Statistically significant difference with $\mathrm{SB}(p<0.05)$. ${ }^{\mathrm{c}}$ Statistically significant difference with BS $(p<0.05)$.

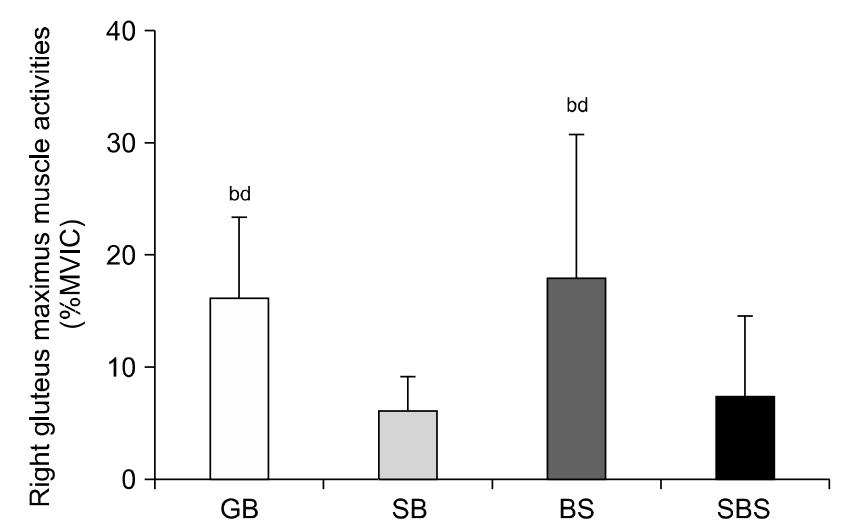

Figure 6. Right gluteus maximus muscle activities. GB: general bridgeexercise, SB: single-legged bridge exercise, BS: bridge exercises with a sling, SBS: single-legged bridge exercise with a sling. ${ }^{b}$ Statistically significant difference with $\mathrm{SB}(p<0.05)$. ${ }^{\mathrm{d}}$ Statistically significant difference with SBS $(\mathrm{p}<0.05)$. trunk were in alignment per bridge condition, muscle activation measurements were taken for 5 seconds $[8,18]$. Data from the first and last second were excluded, while data from the middle three seconds was analysed. After all the conditions were repeated and measured three times and mean values were obtained, values were normalized to MVIC.

Data was collected using a statistical program for Windows IBM SPSS ver. 19.0 (IBM Co., Armonk, NY, USA). Descriptive statistics was used to analyse the general characteristics of subjects, and a one-way repeated measures ANOVA was used to investigate for the effects of various bridge exercise conditions on trunk and lower extremity gluteal muscle activation. To examine for the differences in trunk and lower extremity muscle activation, data was analysed using the least significant difference. The significance level was set at $p<0.05$.

\section{Results}

A comparison of ipsilateral trunk and lower extremity muscle activation according to various bridge conditions

Ipsilateral ES muscle activation was significantly greater in the SB, BS, and SBS conditions compared with the GB condition $(p<0.05)$, and was significantly greater in the SBS condition compared with the SB and BS conditions $(p<0.05)$ (Figure 5).

Ipsilateral GM muscle activation was significantly greater in the GB condition compared with the single-legged and SBS conditions $(p<0.05)$ and was significantly greater in the

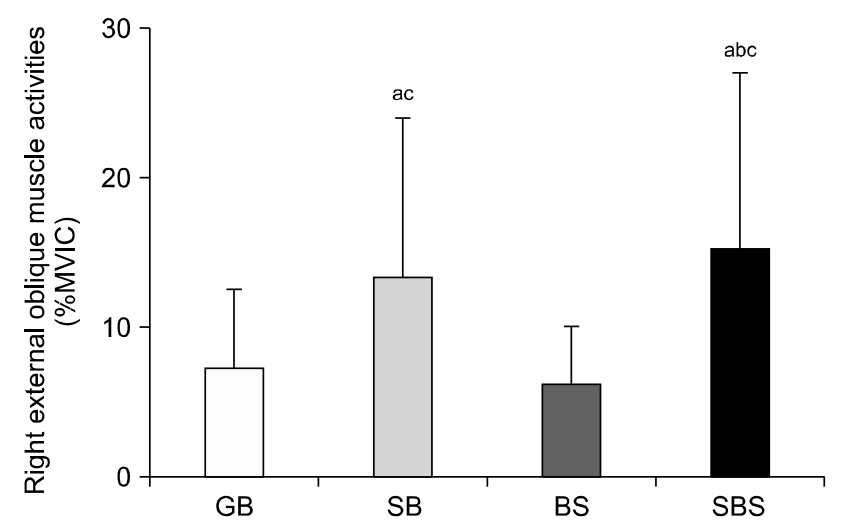

Figure 7. Right external oblique muscle activities. GB: general bridgeexercise, SB: single-legged bridge exercise, BS: bridge exercises with a sling, SBS: single-legged bridge exercise with a

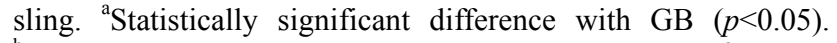
${ }^{\mathrm{b}}$ Statistically significant difference with SB $(p<0.05)$. ' $S$ Statistically significant difference with BS $(p<0.05)$. 
bridge with sling condition compared with SB and SBS conditions $(p<0.05)$ (Figure 6).

Ipsilateral EO muscle activation was significantly greater in the GB, SB, and SBS conditions compared with the BS condition $(p<0.05)$, was significantly greater with the GOBR and SBS compared with the GB condition $(p<0.05)$, and was significantly greater in the SBS condition compared with the SB condition $(p<0.05)$ (Figure 7).

Ipsilateral IO muscle activation was significantly greater in the SB and SBS compared with the GB and BS conditions $(p<0.05)$, and was significantly greater in the SBS compared with the GOBR condition $(p<0.05)$ (Figure 8).

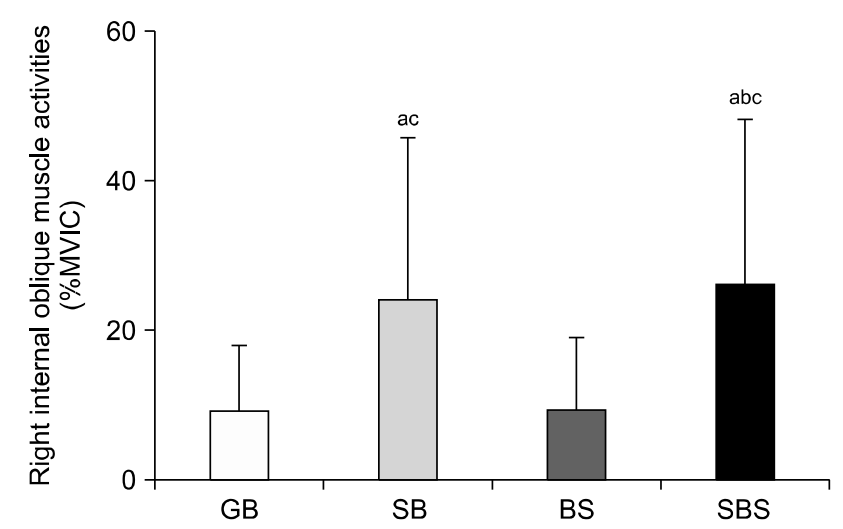

Figure 8. Right internal oblique muscle activities. GB: general bridgeexercise, SB: single-legged bridge exercise, BS: bridge exercises with a sling, SBS: single-legged bridge exercise with a sling. ${ }^{\mathrm{a}}$ Statistically significant difference with GB $(p<0.05)$. ${ }^{\mathrm{b}}$ Statistically significant difference with $\mathrm{SB}(p<0.05)$. ${ }^{\mathrm{c}}$ Statistically significant difference with BS $(p<0.05)$.

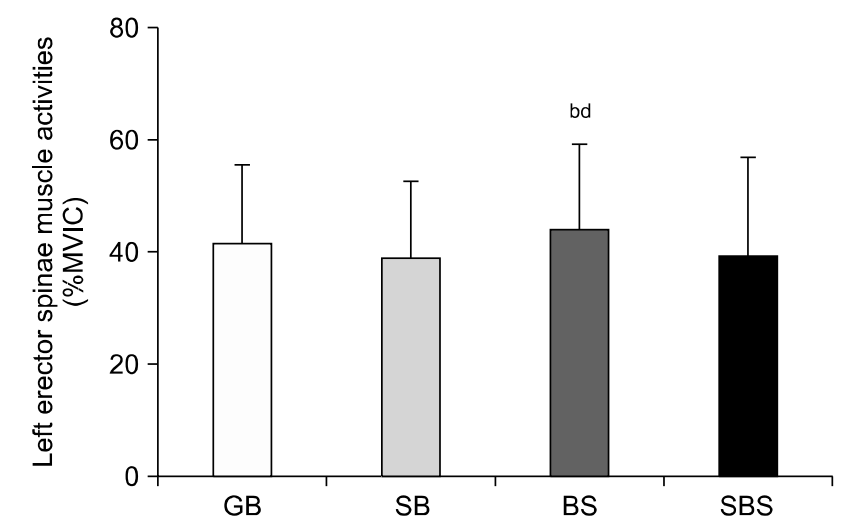

Figure 9. Left erector spinae muscle activities. GB: general bridgeexercise, SB: single-legged bridge exercise, BS: bridge exercises with a sling, SBS: single-legged bridge exercise with a sling. ${ }^{b}$ Statistically significant difference with $\mathrm{SB}(p<0.05)$. ${ }^{\mathrm{d}}$ Statistically significant difference with SBS $(\mathrm{p}<0.05)$.

\section{A comparison of contralateral trunk and lower extremity muscle activation according to various bridge conditions}

Contralateral ES muscle activation was significantly greater in the bridge with sling condition compared with the GB, SB, and SBS conditions $(p<0.05)$ (Figure 9).

Contralateral GM muscle activation was significantly greater in the SB and SBS conditions compared with the GB and $\mathrm{BS}$ conditions $(p<0.05)$, and was significantly greater in the SB condition compared with the SBS condition $(p<0.05)$ (Figure 10).

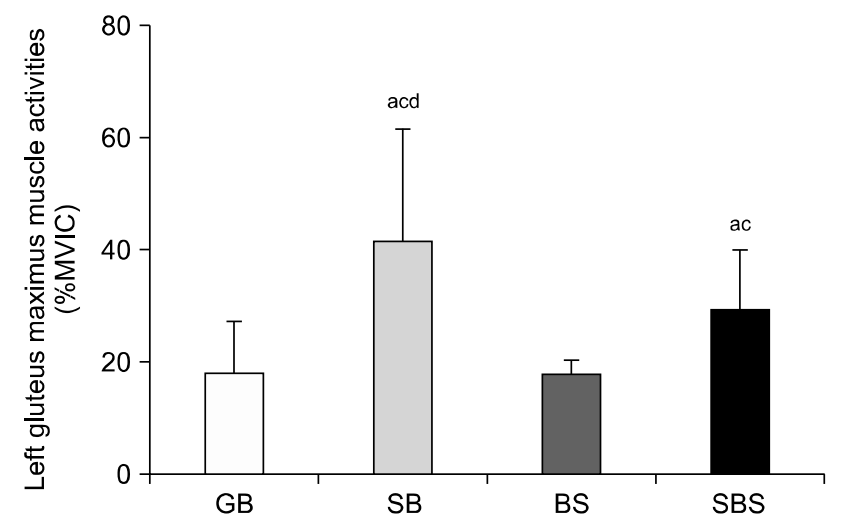

Figure 10. Left gluteus maximus muscle activities. GB: general bridgeexercise, SB: single-legged bridge exercise, BS: bridge exercises with a sling, SBS: single-legged bridge exercise with a sling. " Statistically significant difference with GB $(p<0.05)$. ${ }^{\mathrm{c}}$ Statistically significant difference with BS $(p<0.05)$. ${ }^{\mathrm{d}}$ Statistically significant difference with SBS $(\mathrm{p}<0.05)$.

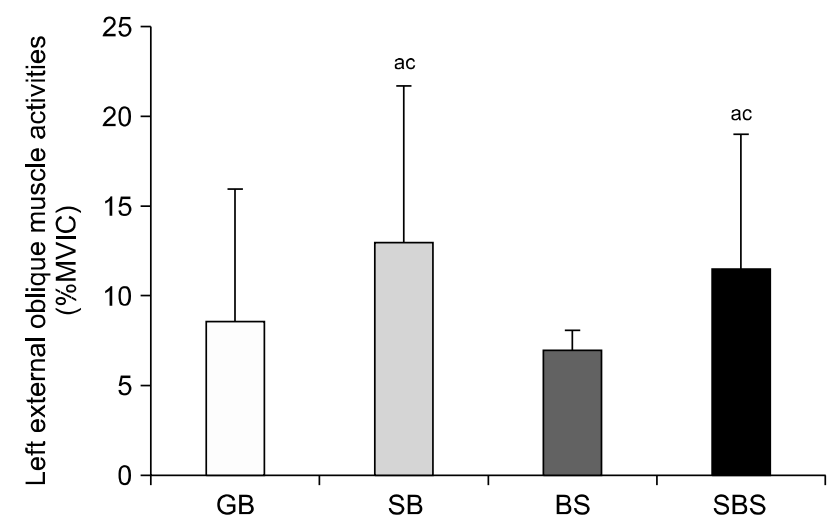

Figure 11. Left external oblique muscle activities. GB: general bridgeexercise, SB: single-legged bridge exercise, BS: bridge exercises with a sling, SBS: single-legged bridge exercise with a sling. ${ }^{a}$ Statistically significant difference with GB $(p<0.05)$. ${ }^{\mathrm{c}}$ Statistically significant difference with BS $(p<0.05)$. 

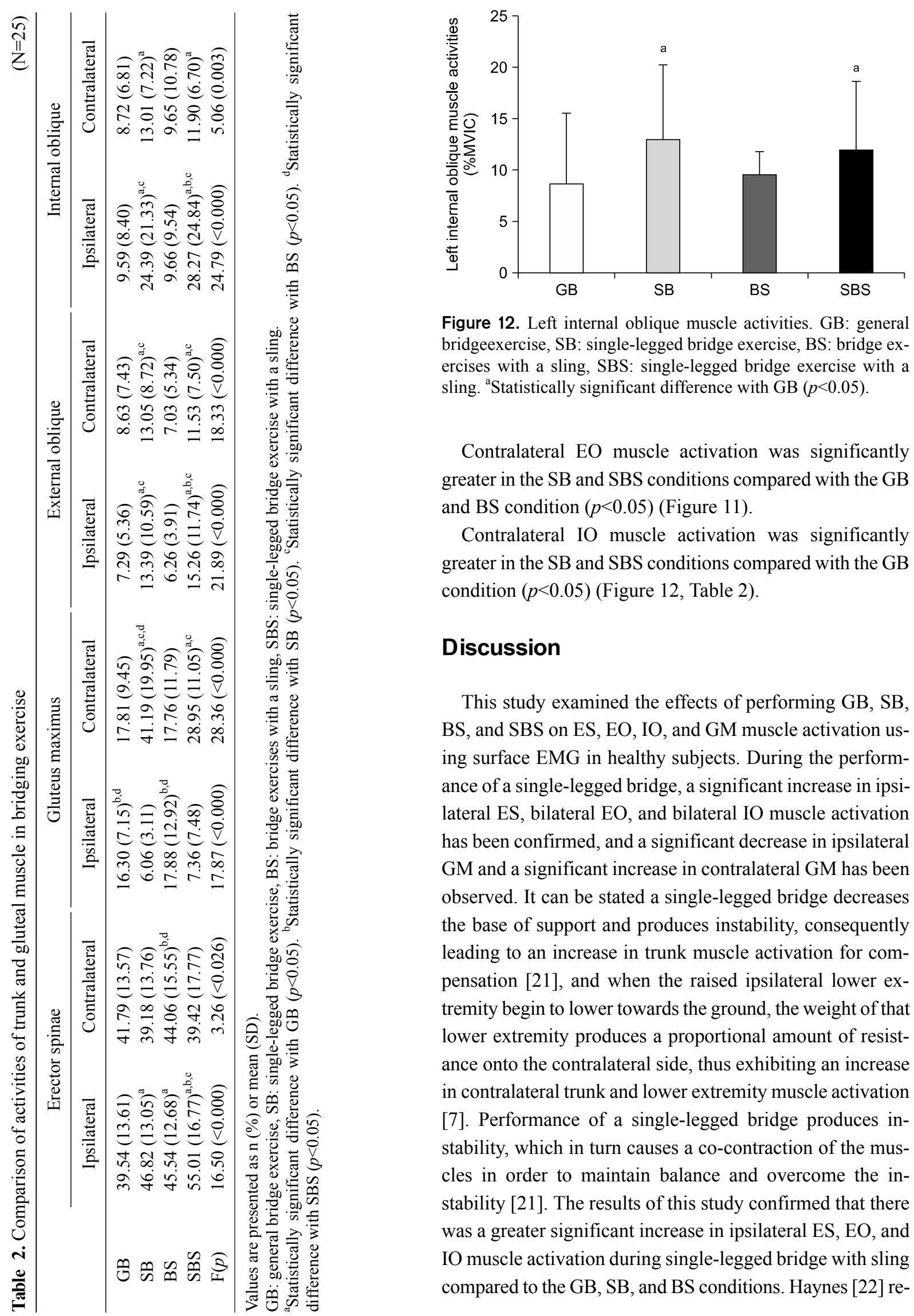

Figure 12. Left internal oblique muscle activities. GB: general bridgeexercise, SB: single-legged bridge exercise, BS: bridge exercises with a sling, SBS: single-legged bridge exercise with a sling. ${ }^{\text {a }}$ Statistically significant difference with GB $(p<0.05)$.

Contralateral EO muscle activation was significantly greater in the SB and SBS conditions compared with the GB and BS condition $(p<0.05)$ (Figure 11).

Contralateral IO muscle activation was significantly greater in the SB and SBS conditions compared with the GB condition $(p<0.05)$ (Figure 12, Table 2).

\section{Discussion}

This study examined the effects of performing GB, SB, $\mathrm{BS}$, and SBS on ES, EO, IO, and GM muscle activation using surface EMG in healthy subjects. During the performance of a single-legged bridge, a significant increase in ipsilateral ES, bilateral EO, and bilateral IO muscle activation has been confirmed, and a significant decrease in ipsilateral GM and a significant increase in contralateral GM has been observed. It can be stated a single-legged bridge decreases the base of support and produces instability, consequently leading to an increase in trunk muscle activation for compensation [21], and when the raised ipsilateral lower extremity begin to lower towards the ground, the weight of that lower extremity produces a proportional amount of resistance onto the contralateral side, thus exhibiting an increase in contralateral trunk and lower extremity muscle activation [7]. Performance of a single-legged bridge produces instability, which in turn causes a co-contraction of the muscles in order to maintain balance and overcome the instability [21]. The results of this study confirmed that there was a greater significant increase in ipsilateral ES, EO, and IO muscle activation during single-legged bridge with sling compared to the GB, SB, and BS conditions. Haynes [22] re- 
ported subjects who participated in various unstable conditions exercises demonstrated remarkable muscle activation on greater instability levels. It can be considered that a greater increase in ipsilateral ES, EO, and IO muscle activation was observed during the SB with use of a sling due to a decrease in stability compared with the performance of a single-legged bridge or without the use of a sling. Bolgla and Uhl [23] reported a greater increase in muscle activation during closed-chain hip abduction exercises in a side-lying position after comparing three closed-chain and three open chained exercises $(p<0.05)$. Lever arm length has a significant role in producing an optimal amount of muscle activation. Increases in lever arm length produces an in crease in mechanical and strength properties of the muscle. It has been observed in this study that there was an increase in ipsilateral muscle activation during the compared with the GB and SB conditions, and placing the contralateral ankle in a sling while using the ipsilateral lower extremity to raise the body produced an increase in lever arm compared with the GB position. The increase in muscle activation is considered to be due to the increase in lever arm.

In this study, a significant increase in ES, EO, IO, and contralateral GM muscle activation has been observed during the performance of a single-legged bridge with sling and single-legged bridge conditions compared with the general bridge condition. Similar results have been found in previous studies. Guthrie et al. [24] did a study on low back pain patients in their twenties where they had performed. A greater muscle activation of the RA has been reported during the performance of a bridge exercise with a sling compared with the $(p<0.05$. Dannelly et al. [16] reported that a gradual application of closed chain exercises in low back pain patients produced an increase in muscle strength and proprioceptive sense. This study had a few limitations. First of all, there was a small number of subjects. Second, all subjects were healthy and thus making it difficult to apply the results to the general population. Since subjects had to perform four types of bridge exercise conditions, it is possible that a learning effect could have occurred. Further studies that will examine changes in muscle thickness, strength and fatigue factors through strength training and its effects on the trunk and lower extremity muscles are warranted.

This study investigated the effects of performing a single-legged bridge with sling exercise on trunk and gluteal muscle activation in healthy subjects. The interventions of this study may be a clinically effective method of treatment, and can be applied to patients with hemiplegia.

\section{Conflict of Interest}

The authors declared no potential conflicts of interest with respect to the authorship and/or publication of this article.

\section{References}

1. Magee DJ. Instability and stabilization: theory and treatment. 2nd Seminar Workbook; 1999.

2. Stevens VK, Bouche KG, Mahieu NN, Coorevits PL, Vanderstraeten GG, Danneels LA. Trunk muscle activity in healthy subjects during bridging stabilization exercises. BMC Musculoskelet Disord 2006;7:75.

3. Richardson CA, Snijders CJ, Hides JA, Damen L, Pas MS, Storm $\mathrm{J}$. The relation between the transversus abdominis muscles, sacroiliac joint mechanics, and low back pain. Spine (Phila Pa 1976) 27:399-405.

4. McGill SM, Cholewicki J. Biomechanical basis for stability: an explanation to enhance clinical utility. J Orthop Sports Phys Ther 31:96-100

5. Handa N, Yamamoto H, Tani T, Kawakami T, Takemasa R. The effect of trunk muscle exercises in patients over 40 years of age with chronic low back pain. J Orthop Sci 2005;5:210-6.

6. Marshall PW, Murphy BA. Core stability exercises on and off a swiss ball. Arch Phys Med Rehabil 2005;86:242-9.

7. Ryerson S, Levit K. Functional movement reeducation: a contemporary model for stroke rehabilitation. New York: Churchill Livingstone; 1997.

8. O'Sullivan SB, Schmitz TJ, Fulk G. Physical rehabilitation. 6th ed. Danvers: FA Davis; 2013.

9. Kisner C, Colby LA. Therapeutic exercise: foundations and techniques. 6th ed. Philadelphia: FA Davis; 2012.

10. O'Sullivan PB. Lumbar segmental 'instability': clinical presentation and specific stabilizing exercise management. Man Ther 2000;5:2-12.

11. Kisner C, Colby LA. Therapeutic exercise: foundations and techniques. 4th ed. Philadelphia: FA Davis; 2002.

12. Maeo S, Chou T, Yamamoto M, Kanehisa H. Muscular activities during sling- and ground-based push-up exercise. BMC Res Notes 2014;7:192.

13. Escamilla RF, Lewis C, Bell D, Bramblet G, Daffron J, Lambert $\mathrm{S}$, et al. Core muscle activation during swiss ball and traditional abdominal exercises. J Orthop Sports Phys Ther 2010;40:265-76.

14. Imai A, Kaneoka K, Okubo Y, Shiina I, Tatsumura M, Izumi S, et al. Trunk muscle activity during lumbar stabilization exercises on both a stable and unstable surface. J Orthop Sports Phys Ther 2010;40:369-75.

15. Lehman GJ, Gilas D, Patel U. An unstable support surface does not increase scapulothoracic stabilizing muscle activity during push up and push up plus exercises. Man Ther 2007;13:500-6.

16. Dannelly BD, Otey SC, Croy T, Harrison B, Rynders CA, Hertel $\mathrm{JN}$, et al. The effectiveness of traditional and sling exercise strength training in women. J Strength Cond Res 2011;25:464-71.

17. Kang H, Jung J, Yu J. Comparison of trunk muscle activity during bridging exercises using a sling in patients with low back 
pain. J Sports Sci Med 2012;11:510-5.

18. Stevens VK, Coorevits PL, Bouche KG, Mahieu NN, Vanderstraeten GG, Danneels LA. The influence of specific training on trunk muscle recruitment patterns in healthy subjects during stabilization exercises. Man Ther 2007;12:271-9.

19. Criswell E. Cram's introduction to surface electromyography. Sudbury: Jones \& Bartlett Publishers; 2010.

20. Kendall FP, McCreary EK, Rodgers MM, Provance PG; et al. Muscles: testing and function with posture and pain. 5th ed. Baltimore: Lippincott Williams \& Wilkins; 2005.

21. Shumway-Cook A, Woollacott M. Motor control: translation re- search into clinical practice. 3rd ed. Baltimore: Lippincott Williams \& Wilkins; 2001. p. 158-86.

22. Haynes W. Core stability and the unstable platform device. J Bodyw Mov Ther 2004;8:88-103.

23. Bolgla LA, Uhl TL. Electromyographic analysis of hip rehabilitation exercises in a group of healthy subjects. J Orthop Sports Phys Ther 2005;35:487-94.

24. Guthrie RJ, Grindstaff TL, Croy T, Ingersoll CD, Saliba SA. The effect of traditional bridging or suspension-exercise bridging on lateral abdominal thickness in individuals with low back pain. $\mathbf{J}$ Sport Rehabil 2012;21:151-60. 